\title{
Complejidad y estandarización en las bóvedas tardogóticas
}

\author{
José Carlos Palacios Gonzalo \\ y Rafael Martín TALAVERANO \\ Escuela Técnica Superior de Arquitectura \\ Universidad Politécnica de Madrid \\ josecarlos.palacios@upm.es \\ r.martin@upm.es
}

\begin{abstract}
RESUMEN
El principio de complejidad como vector de evolución de la arquitectura gótica fue una idea ampliamente desarrollada por Paul Frankl. Este desarrollo constatado hacia una sofisticación y multiplicación de los elementos en las bóvedas de crucería bajomedievales, fue sin embargo posible gracias al empleo de avanzados recursos geométricos que tenían como objetivo aumentar la estandarización y simplificación de los procesos constructivos. De este modo, al mismo tiempo que los diseños de las bóvedas adquirían caracteres más complejos y elaborados, se desarrollaban técnicas que permitían facilitar el trazado y la construcción de las mismas. El presente texto pretende poner de manifiesto alguno de estos recursos detectados en el análisis de varios casos particulares de bóvedas de crucería bajomedievales. Como se puede observar, el sofisticado manejo de los recursos geométricos y los notables avances tecnológicos que se produjeron ponen de manifiesto que los abovedamientos bajomedievales constituyen una de las páginas más brillantes de la historia de la construcción europea.
\end{abstract}

Palabras clave: arquitectura gótica, bóvedas de crucería, geometría, proceso constructivo.

\begin{abstract}
The idea that the gothic architecture followed a rising complexity level as an aesthetical principle was deeply developed by Paul Frankl. This evolution searching more complicated designs was made possible by using advanced geometrical methods that tried to simplify the construction process. In other words, while the amount of ribs was getting bigger and the designs were more elaborated, a search for standardization was developed. The main objective of this paper is to focus on those geometrical methods through the analysis of some particular late gothic ribbed vaults. The technological advances that are analyzed became a specific feature of one of the most important chapters of the European construction history.
\end{abstract}

Keywords: gothic architecture, ribbed vaults, geometrical methods, technological advances.

\section{Introducción}

\section{Evolución de la arquitectura gótica}

El principio de complejidad como vector de evolución de la arquitectura gótica fue una idea ampliamente desarrollada por Paul Frank $1^{1}$. Este desarrollo constatado

\footnotetext{
1 P. FRANKL, Arquitectura gótica, Madrid, 2002.
} 
hacia una sofisticación y multiplicación de los elementos en las bóvedas de crucería bajomedievales, fue sin embargo posible gracias al empleo de avanzados recursos geométricos que tenían como objetivo aumentar la estandarización y simplificación de los procesos constructivos. En concreto, la búsqueda de trazados geométricos que posibilitasen el empleo de un mínimo número de curvaturas es una característica que puede observarse tanto en ejemplos hispanos como europeos ${ }^{2}$. Las ventajas que se obtienen de igualar los radios de curvatura de los arcos de una bóveda son evidentes: todas las dovelas con las que se construye la nervadura son iguales y las cimbras de madera que ayudarán a su construcción serán idénticas y, por lo tanto, reutilizables. El presente texto pretende poner de manifiesto alguno de estos recursos detectados en el análisis de varios casos particulares de bóvedas de crucería bajomedievales. En ellos, la elevada complejidad de sus diseños se resuelve con criterios de estandarización que permiten simplificar en cierta medida el proceso constructivo.

\section{Del arco de medio punto al arco ojival}

Uno de los rasgos distintivos que caracterizan a la arquitectura gótica desde sus comienzos es el empleo del arco apuntado frente al uso exclusivo del arco de medio punto en épocas anteriores. El uso generalizado del arco ojival, más allá de constituir un rasgo estilístico fundamental, supone una evolución tecnológica notable. Si se pretende salvar una luz determinada con un arco semicircular, la solución es única, las dovelas que constituyen ese arco vendrán determinadas por el radio de curvatura de la circunferencia que describe el arco. Si, en la misma obra, se requiere otro arco con una luz distinta, las dovelas necesarias para este arco tendrían que tener otro radio de curvatura diferente y, por tanto serían distintas a la del arco anterior y, del mismo modo, ocurriría con todos los arcos de luces distintas. Por tanto, cuando se construye con arcos de medio punto, la organización, almacenamiento y montaje de las dovelas se complica extraordinariamente, ya que las piezas no pueden intercambiarse entre si; cada una requiere su ubicación adecuada.

Sin embargo, si para salvar la misma luz, en vez de un arco semicircular, se optase por construir un arco apuntado la solución es indeterminada, es decir, existen infinitos arcos ojivales que pueden cubrir una determinada luz. Por ello es necesario definir algún otro parámetro que fije la solución a emplear. El parámetro más usual que se manejaban en la época pleno y bajomedieval era la altura de la clave (flecha del arco); este dato determinaba el radio y el centro de curvatura, suponiendo que el arco en su inicio parte con tangencia vertical. Este grado de indeterminación inherente a la geometría del arco ojival supone a la vez una de sus ventajas más potentes. Si se pretende construir otro arco que cubra una luz distinta, es posi-

\footnotetext{
2 W. MÜLLER, Grundlagen gotischer bautechnik: ars sine scientia nihil est, München, 1990.
} 

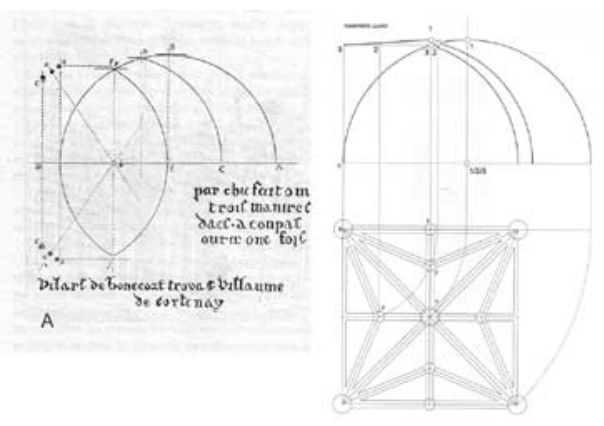

Fig. 1. Interpretación de Viollet-le Duc del dibujo de Villard de Honnecourt (A) donde se muestra cómo todos los arcos de una bóveda pueden trazarse a partir de una semicircunferencia. B, traza de una bóveda de cinco claves a partir de la semicircunferencia del arco diagonal, el resultado es una bóveda con su caballete casi horizontal.

curvaturas. Por tanto, el empleo del arco ojival supone una ventaja notable de cara a la organización de la obra y la transmisión de las instrucciones entre los distintos miembros del taller que construye el edificio (fig. 1).

\section{Estandarización de las bóvedas bajomedievales}

La evolución de la arquitectura gótica hispana en plena transición del mundo bajomedieval a la época moderna, viene marcada por el desarrollo de bóvedas de crucería cada vez más complejas en las que los nervios y las claves que las componen se multiplican incesantemente ${ }^{3}$. El modelo más frecuente es la bóveda de crucería estrellada, formada por cinco claves (una central y cuatro secundarias). Esta composición sirve como base a una gran cantidad de composiciones más complejas en las que tanto las claves como los terceletes se van incrementando. Por regla general todas estas bóvedas usan arcos trazados a compás, es decir con un solo centro situado en la línea de imposta de la bóveda, pero también son muy frecuentes las bóvedas trazadas con arcos de tres centros, es decir, óvalos. Las estrategias geométricas detectadas en ejemplos construidos, que se usan para lograr que todos los arcos con los que va a construirse la bóveda puedan llegar a ser iguales, son los siguientes (fig. 2).

A.-Arcos de una curvatura: igualación con el nervio diagonal. Todos los arcos son iguales porque coinciden con la curvatura del nervio diagonal.

3 J. GÓMEZ MARTÍNEZ, El gótico español en la Edad Moderna: bóvedas de crucería, Valladolid, 1998 


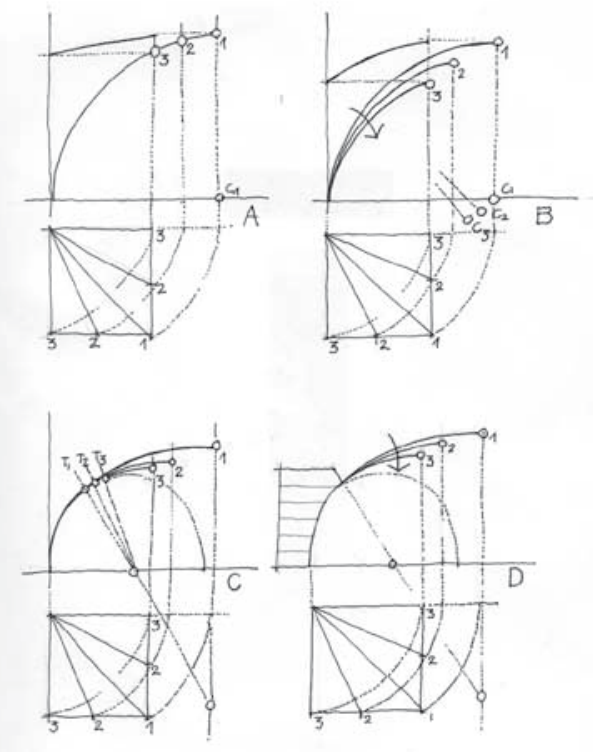

Fig. 2. Resumen de los cuatro recursos detectados en las bóvedas góticas españolas destinados a estandarizar las curvaturas de sus crucerías.

B.-Arcos de una curvatura: inclinación de los nervios. Los arcos son iguales porque el arco diagonal (semicircular) se va inclinando hacia delante hasta alcanzar las alturas de las claves secundarias previamente determinadas.

C.-Arcos de doble curvatura: deslizamiento de la parte superior. El punto de tangencia entre las dos curvaturas del óvalo se desplaza pero todos los arcos se pueden construir con las mismas curvaturas.

D.-Arcos de doble curvatura: inclinación de la parte superior. Si se considera que la parte inferir del arco oval en realidad no es un nervio, sino que está constituido por piezas completas solidarias con el muro (jarjas), la parte superior del óvalo puede comenzar siempre a la misma altura, allí donde acaba la jarja. Desde este punto, como en el caso B, el arco que forma la parte superior del óvalo diagonal se va inclinando hasta alcanzar las alturas de la claves.

\section{Bóvedas en la que todos sus arcos tienen la misma curvatura}

Cuando la bóveda es de planta cuadrada, si se trazan los arcos diagonales semicirculares y, a continuación, con el mismo radio de curvatura se trazan los cuatro arcos perimetrales, las claves de estos arcos alcanzan una altura ligeramente inferior a la clave central. Se trata, por tanto, de una bóveda de rampante prácticamente horizontal en la que los ojivos y los formeros son arcos de igual curvatura. Además, si se trazan los terceletes con el mismo radio, conseguimos construir una bóveda en la que todos los arcos tienen la misma curvatura (fig. 1). Esta particularidad, que tanto puede simplificar la talla de dovelas y la confección de cimbras, hizo muy frecuente este tipo de bóvedas.

Veamos la aplicación de este principio en la bóveda que cubre los brazos del crucero de la catedral de Astorga. Esta bóveda atribuida a Rodrigo Gil debió llevarse a cabo entre 1550 y 1570 . La bóveda tiene una planta cuadrada de 7,58 metros de lado y su clave central alcanza una altura de 5,14 metros sobre el plano de impostas. Tiene una pareja de terceletes en cada dirección y los combados describen dos figuras tangentes entre sí: cuatro lóbulos formados por circunferencia secantes en el centro y un cuadrilóbulo convexo, es decir, con su curvatura al exterior, aca- 
bados en conopios. Este diseño podría considerarse como característico de los Hontañón y, con más o menos variantes, fue repetido en multitud de iglesias castellanas (fig. 3).

Como puede apreciarse en la figura 3A, los terceletes se sitúan en la bisectriz del ángulo que forman la diagonal y el formero; el resto de las claves se obtiene superponiendo sobre la planta una trama de $4 \times 4$. Esta retícula fija el punto 1, con el que los lóbulos centrales queda determinados, así como el punto de tangencia 4. Con la misma circunferencia con que

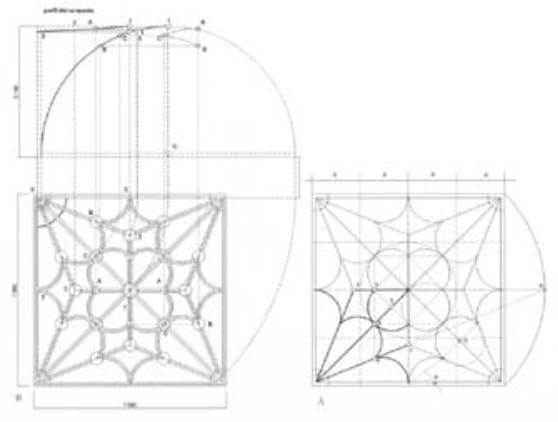

Fig. 3. Catedral de Astorga, bóvedas sobre las capillas de los cruceros; A, traza de la planta y B, de la curvatura de sus arcos.

se dibuja el lóbulo central se traza la que tiene su centro en 5. Al llevar a cabo la reconstrucción geométrica de las curvaturas de los arcos en la figura 3B, se observa que el arco diagonal es una circunferencia perfecta peraltada $15 \mathrm{~cm}$. en el arranque. Con las medidas reales de la bóveda se reconstruyen sus trazas y se observa que las alturas de las claves de los terceletes 2 y los arcos formeros 3 vienen a coincidir en la trayectoria del arco diagonal (puntos 2 y 3). Lo mismo sucede con las claves secundarias A, B y C. El resultado es una bóveda en la que sus caballetes, los rampantes, son prácticamente horizontales, apenas una ligera inclinación desde la clave central 1 hasta las claves de los formeros 3. Por tanto, los arcos formeros y terceletes son arcos apuntados que se forman con porciones de la semicircunferencia del crucero.

\section{Bóvedas con los arcos inclinados}

En el ángulo noroeste del claustro de la catedral de Segovia, se encuentra una bóveda trazada por Juan Guas, que destaca por la originalidad y singularidad de su composición. ${ }^{4}$ A primera vista destaca la falta de simetría en todos sus planos, de modo que ésta sólo existe en los planos verticales que contienen a los arcos diagonales. Además, no existe clave central, puesto que los arcos ojivos se "desvían" para componer un rombo central, en el cual sí se han tallado a modo de pliegues de la plementería las líneas geométricas por donde deberían continuar los nervios. Este trazado tan singular, se puede observar en algunas fuentes medievales del ámbito germano, como en la colección de dibujos Wiener Sammlungen o en el Cuaderno de Frankfurt ${ }^{5}$.

4 J.M. MERINO DE CÁCERES, El claustro de la catedral de Segovia, Segovia, 1996.

5 H.J. BÖKER, Architektur der Gotik: Bestandskatalog der weltgrößten Sammlung an gotischen Baurissen (Legat Franz Jäger), München, 2005; y F. BUCHER, Architector. The lodge books and sketch books of medieval architects, New York, 1979. 
Sin embargo, una vez más, la versatilidad del sistema de trazado de las bóvedas de crucería permite hallar la montea de esta composición sin problemas (fig. 4). Aunque materialmente no se encuentren completos, los arcos ojivos forman un semicírculo que determina la altura del punto central de la bóveda. Una vez trazados, el arquitecto define la forma que ha de tener la bóveda, que queda determinada por sus dos secciones transversales, la longitudinal y la transversal. La línea que dibuja el caballete de la bóveda recibía el nombre de rampante; por tanto, las líneas de los rampantes definen la "caída" de la superficie abovedada hacia los arcos formeros y perpiaños y, además, determinan la atura de las claves que se encuentran sobre ellos.

Con estas premisas, los arcos ojivos, terceletes y formeros deberían ser distintos entre sí, cada uno con su particular

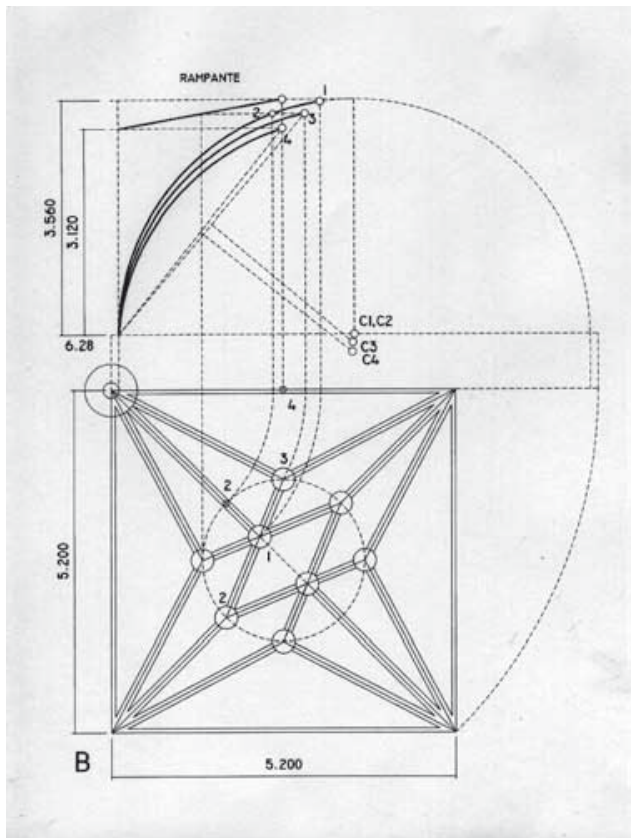

Fig. 4. Catedral de Segovia, bóveda del claustro. La bóveda está construida con arcos coincidente en su curvatura e inclinados. curvatura. Sin embargo, un examen más detallado desvela unos recursos singulares para conseguir que todos los nervios puedan trazarse con una única curvatura. En primer lugar, el radio del arco ojivo queda determinado por la semidiagonal del cuadrado (ya que este arco es una semicircunferencia), y del mismo modo la altura de la clave central 1. Llega el turno de trazar el tercelete, punto 3, situado a la altura que quedó fijada con el rampante; para trazar este arco, se usa el mismo arco ojivo, basta con hacerlo bascular hacia delante hasta que alcance la altura 3. Es evidente que este arco tendrá su centro bajo la línea de impostas y, en su origen, no parte tangente a la línea vertical, forma un pequeño quiebro en el arranque que, en realidad, pasa prácticamente desapercibido. Con el arco formero puede procederse de la misma forma, de nuevo, desde el origen volvemos a pivotar el arco diagonal hasta que alcance la altura 4; de nuevo el arco parte inclinado respecto a la vertical y su centro se encuentra bajo la línea de impostas.

Por tanto, como resumen, podríamos decir que este método de estandarizar curvaturas resulta opuesto al descrito anteriormente. En el primer caso, la forma de la bóveda era consecuencia de usar el mismo arco diagonal para trazar los demás arcos, la consecuencia era invariablemente una bóveda de rampante llano. Con el método que acabamos de describir, se determina en primer lugar la forma de la bóveda, y ésta podrá adaptarse a superficies previamente definidas. Posteriormen- 


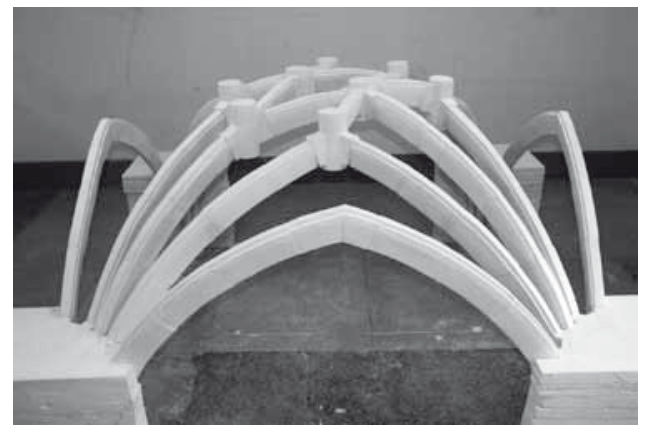

Fig. 5. Maqueta realizada en la escuela de Arquitectura de Madrid a escala 1:3 de la bóveda de Juan Guas. te, al dibujar sus rampantes se obtendrán las alturas de sus claves principales. Finalmente, basta con ir inclinando el arco diagonal par alcanzar la altura de dichas claves. En el Departamento de Construcción de la Escuela de Arquitectura de Madrid hubo ocasión de verificar las hipótesis anteriormente expuestas mediante la construcción de una maqueta a escala 1:3 de la bóveda de Juan Guas (fig. 5). Esta experiencia confirma con rotundidad la validez de este recurso constructivo.

Las consecuencias de estos hábiles recursos son evidentes: todos los arcos tienen la misma curvatura, o lo que es lo mismo, toda la bóveda se construye con un solo arco, por lo que todas las dovelas son iguales. No es necesario insistir en las ventajas para la organización y ejecución de la obra que esto supone. La búsqueda de la estandarización y sistematización se demuestra claramente en este caso, aunque el diseño geométrico sea verdaderamente sofisticado y singular.

\section{Bóvedas con arcos ovales}

Entre las nuevas bóvedas que se desarrollan en el bajo gótico hispano se encuentran las de crucería oval ${ }^{6}$. Son bóvedas en las que los arcos diagonales, en lugar de semicircunferencias, son arcos de tres centros (óvalos). En principio, podrían calificarse como bóvedas rebajadas, aunque en ocasiones, en sus arranques, pueden peraltarse y alcanzar una altura en la clave central similar al de una bóveda normal. En este tipo de bóvedas, solamente los arcos diagonales y los perpiaños son óvalos completos, las curvaturas del resto de los arcos (terceletes y formeros), suelen ser una porción de la mitad del arco oval que describe la diagonal, así que, por simetría, estos arcos son arcos apuntados de cuatro centros: arcos Tudor.

Tienen algunas ventajas indudables. En primer lugar, como acabamos de mencionar, son bóvedas rebajadas, lo cual significa que al alcanzar menos altura que una bóveda tradicional, permite limitar la altura general del edificio y con ello su coste. En segundo lugar, son bóvedas de superficie relativamente plana en su coronación, lo que posibilita determinados diseños de nervios más complejos, de difícil ejecución sobre superficies curvas más acentuadas. Además, el uso de arcos ovales permite estandarizar en gran medida la traza de los nervios ya que, como pone de

6 J.C. PALACIOS GONZALO, La cantería medieval, la construcción de la bóveda gótica española, Madrid, 2009. 


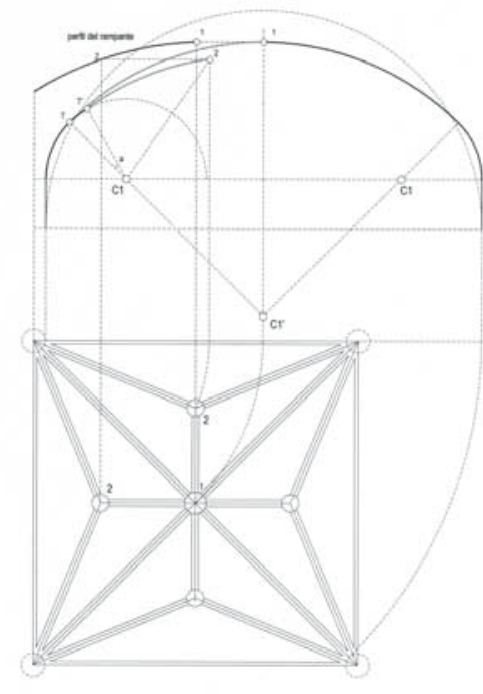

Fig. 6. Los nervios de una bóveda oval se pueden estandarizar usando siempre las mismas curvaturas; en la figura los terceletes y el arco diagonal se forman con los mismos arcos.

(2), como puede verse en la figura 7A, se sitúan en la posición de la diagonal, entre el crucero y el formero; ya conocemos la forma en la que se fijaba la posición de esta clave mediante la circunferencia circunscrita y la recta 1-0 que, al cruzarse con el eje, determina el punto 2. La bóveda, en su centro, luce un combado circular cuyo diámetro lo determina la longitud 2-3, es decir, la distancia que separa la clave de tercelete y la del formero. Por último, tiene en las esquinas cuatro combados en cuarto de circunferencia cuya medida parece venir determinada por la alineación A-B-C; es éste un combado abierto, es decir, desti- manifiesto la figura 6, una vez trazado el óvalo de la diagonal con sus centro $\mathrm{C} 1$ y C1', el tercelete 2 se puede trazar con las mismas curvaturas: la inferior, con centro en 1, y la superior, haciendo rodar la curvatura superior del ovalo desde el punto de tangencia t hasta el t'. Este ingenioso uso de los dos fragmentos del óvalo permite que, en ocasiones, con las dos curvas que forman el óvalo diagonal se pueda resolver el resto de las nervaduras que conforman la bóveda.

Veamos un caso de este tipo de bóvedas realizado por uno de los arquitectos bajomedievales más creativos y sorprendentes del gótico español. Se trata de la bóveda que el arquitecto Juan de Álava construyó en el claustro del monasterio de San Esteban, en Salamanca hacia 1533 (fig. 7) ${ }^{7}$.

La bóveda tiene planta cuadrada y consta de cinco claves. Los terceletes

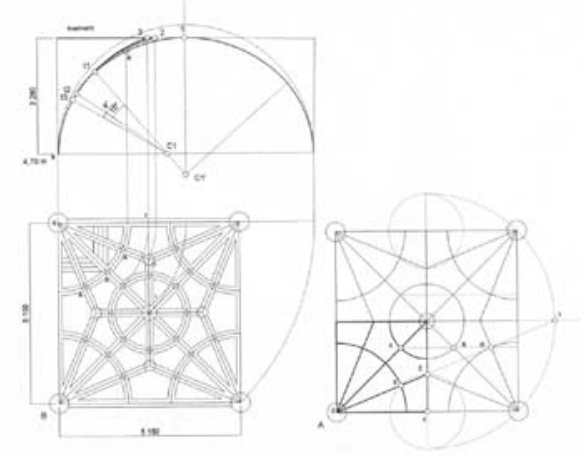

Fig. 7. Convento de San Esteban, Salamanca, claustro. Trazas de la planta (A) y de la curvatura de sus $\operatorname{arcos}(\mathrm{B})$. Obsérvese el arco oval diagonal y cómo se obtiene el rampante horizontal deslizando la parte superior del óvalo sobre la inferior. Los arcos son todos iguales.

\section{A. CASTRO SANTAMARÍA, Juan de Álava, arquitecto del Renacimiento, Salamanca, 2002.}


nado a conectarse con el de la bóveda contigua y a producir un encadenamiento de nervaduras muy característico de este arquitecto.

Al analizar la traza de los arcos (fig. 7B), constatamos inmediatamente que la clave del arco diagonal queda por debajo del arco de medio punto teórico; este arco es, por tanto, un arco rebajado que ha de trazarse con tres centros. Con las medidas que se han tomado in situ, se ha trazado el óvalo 1, de centros $\mathrm{C} 1$ y $\mathrm{C} 1$ ', que parece ajustarse fielmente al arco real.

A continuación pasamos a trazar los terceletes, teniendo en cuenta que, imperativamente, deben alcanzar la altura que determina el rampante horizontal de esta bóveda. Es evidente que el tercelete y el formero podrían ser arcos apuntados diferentes entre sí, pero es mucho más ventajoso construirlos con el mismo nervio diagonal, y conseguir con ello que los tres arcos que constituyen esta bóveda tengan sus radios de curvatura iguales. Para llevar a cabo esta operación, basta con hacer rodar el tramo superior del arco 1 , sobre su parte inferior $15^{\circ}$ aproximadamente; esta operación hace que la clave 1 se traslade a la posición 2 , y el punto de tangencia del óvalo 1 pase a 2 . La clave del formero 3, se lleva a cabo haciendo rodar la parte superior del óvalo 4 sobre la parte inferior. De esta forma se obtienen tres arcos diferentes, pero que, sin embargo, tienen en común la parte inferior y la superior.

La bóveda, al ser rebajada, presenta una cumbrera achatada que permite colocar la rosca circular en su cúspide logrando que este combado describa su circunferencia sin excesivas fragmentaciones. Lo mismo sucede con el combado de esquina que, gracias a que los tres arcos de la bóveda son el mismo óvalo, puede describir una circunferencia continua y horizontal.

\section{Bóvedas con arcos ovales inclinados}

Para ilustrar los principios geométricos de esta traza examinaremos la bóveda del sotocoro del convento de San Esteban de Salamanca, también construida por el arquitecto Juan de Álava hacia 1520 (fig. 8). Se trata, de una bóveda fuertemente rebajada, una bóveda plana lograda gracias al uso de arcos ovales de muy poca alzada. Su planta es rectangular, y sus dimensiones aproximadas son de $15 \times 7,5$ metros. Para el rampante longitudinal (en la dirección del eje de la nave), Juan de Álava determina una línea recta y horizontal, es decir, la altura de la clave central y la del arco perpiaño ha de ser la misma; por el contrario, en la dirección transversal fija un rampante ligeramen-

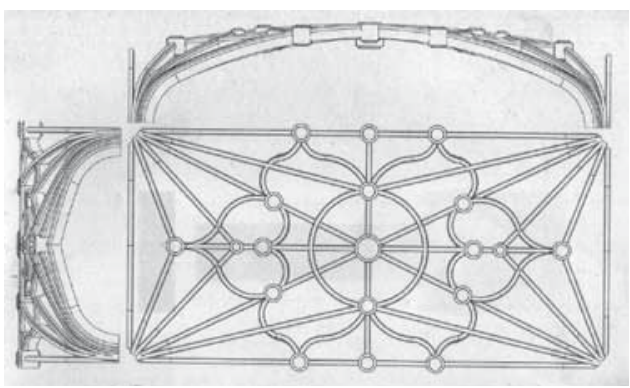

Fig. 8. Planta y secciones de la bóveda del sotocoro del convento de San Esteban mostrando su sección longitudinal horizontal y su sección transversa curvada e inclinada hacia el formero. 
te curvado con caída hacia la clave del arco formero. Estas dos líneas son fundamentales ya que definen con precisión la forma de la bóveda y, además, fijan la altura de las claves secundarias.

Para determinar la curvatura de los terceletes, el arquitecto parece haber usado la estrategia que se resume en el croquis que acompaña a la figura 9. En primer lugar, la parte inferior del óvalo, que no es un nervio individualizado ya que coincide exactamente con el jarjamento de la bóveda, se lleva a cabo mediante lechos horizontales. Estas hiladas horizontales alcanzan la altura del punto de tangencia entre la curvatura superior e inferior del óvalo diagonal. Este óvalo se había trazado con la altura de la clave central (punto 1) que el arquitecto esti-

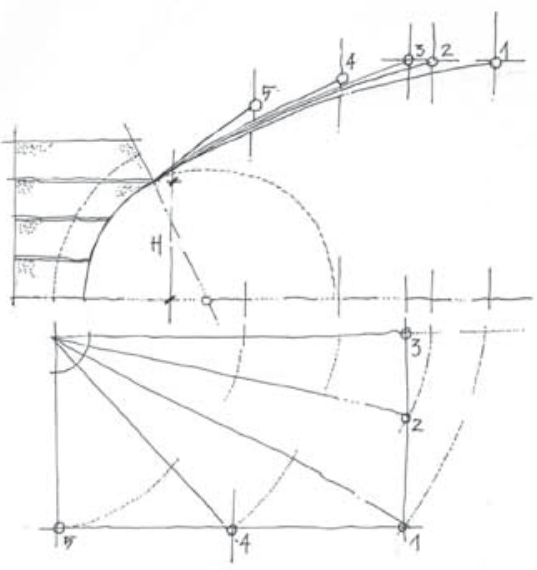

Fig. 9. Croquis en que se muestra la estrategia seguida por el arquitecto para estandarizar las curvaturas de los arcos. Toda la bóveda se traza con la parte superior del óvalo diagonal. mó oportuna. En segundo lugar, se determina que todos los terceletes han de ser arcos ovales con idénticas curvaturas que el arco diagonal. A continuación, se fijan dos condiciones que van a simplificar extraordinariamente la construcción de la bóveda. Por un lado, se hace coincidir la parte inferior del óvalo de los terceletes con el del arco diagonal; por otro lado, se determina que la altura de la jarja que fijó el arco diagonal $\mathrm{H}$, sea idéntica para todos los arcos. Con estas condiciones, los distintos terceletes se trazan fácilmente, haciendo rotar desde el punto $\mathrm{H}$ la parte superior del arco diagonal hasta que por el otro extremo coincida con la altura de cada una de las claves. En definitiva, la bóveda ha sido construida con un solo arco: la parte superior del óvalo del arco diagonal.

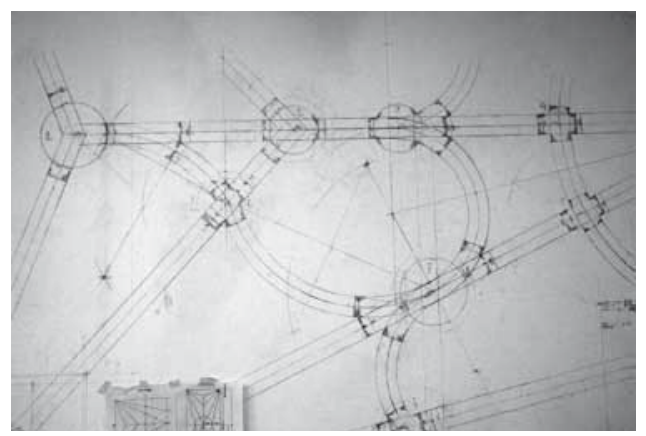

Fig. 10. Escuela de Arquitectura de Madrid. Trazas a tamaño natural destinadas a la construcción de un modelo reducido de la bóveda a escala 1:3.
En la Escuela de Arquitectura de Madrid, hubo ocasión de comprobar las hipótesis expuestas anteriormente mediante la construcción real de una maqueta de esta bóveda. Para la realización de la bóveda, se procedió, en primer lugar, a dibujar sus monteas, es decir sus trazas a tamaño real, en planta y alzado. Siguiendo las pautas que conocemos de los talleres de cantería medievales, de estos dibujos se fueron extrayendo los datos que permitieron acometer la talla de cada una de las 
piezas que componen la bóveda (fig. 10). Una vez que el proceso de talla hubo terminado, se procedió al montaje de la bóveda, colocando sobre cimbras de madera las piezas previamente talladas. Al igualar las curvaturas de los arcos, también las cimbras se ven en extremo simplificadas y pueden ser reutilizadas, optimizándose el proceso constructivo. El resultado final es un modelo reducido de la bóveda que confirma las hipótesis anteriormente

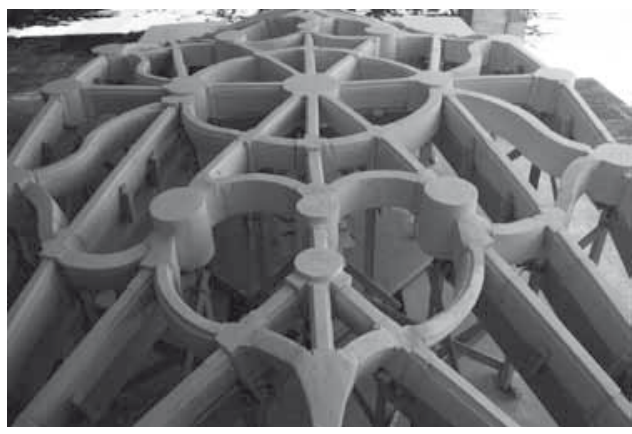

Fig. 11. La reproducción de la complicada bóveda de Juan de Álava construida con un solo arco. expuestas (fig. 11).

\section{Bóvedas centroeuropeas}

La gran revolución que supuso el desarrollo de la bóveda de crucería tardogótica hispana tiene su origen directo en la llegada de una serie de maestros centroeuropeos a los focos burgalés y toledano. Si bien el impulso que supuso esta influencia en el desarrollo de los abovedamientos hispanos fue muy grande y notable, éstos no llegaron a alcanzar la brillantez y virtuosismo de los diseños del ámbito germano, donde los recursos geométricos empleados son de una gran complejidad y eficacia. Como contrapunto a la exposición de los análisis de las bóvedas hispanas, se presenta un caso paradigmático de abovedamiento germano, con uno de los métodos de trazado más empleados para lograr una sistematización y optimización de la ejecución: el prinzipalbogen, o arco principal.

Se ha realizado un análisis geométrico de los nervios de la bóveda del palacio Albrechtsburg, en Meissen (Alemania), que es un ejemplo de las llamadas schlin-

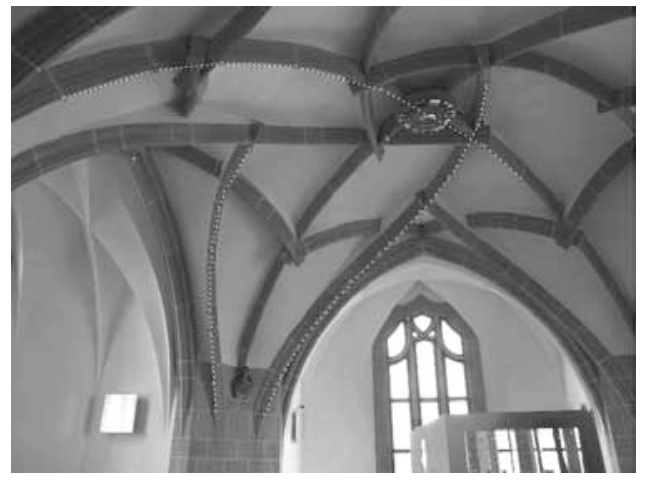

Fig. 12. Nervios analizados de la bóveda del palacio Albrechtsburg (Meissen, Alemania). grippengewölbe (fig. 12). Algunos de sus nervios están constituidos por curvas planas (tienen una proyección horizontal que es una línea recta), similares a los vistos hasta ahora; sin embargo, la mayor parte de ellos son curvas tridimensionales que no quedan definidas únicamente por su proyección horizontal. Tampoco se puede obtener una proyección vertical que aporte su verdadera magnitud, por lo que es necesario algún método sistemático y sencillo de trazado de estas líneas aparentemente carentes de razón geométrica alguna. 
Las fuentes originales mencionan el sistema denominado prinzipalbogen, o arco principal ${ }^{8}$. Según este método, todos los nervios de la bóveda se generan a partir de un único arco de circunferencia: la distancia que se recorre en planta entre dos puntos de un nervio cualquiera se traslada a un plano vertical donde se ha dibujado el arco principal, de modo que la diferencia de altura entre esos dos puntos es

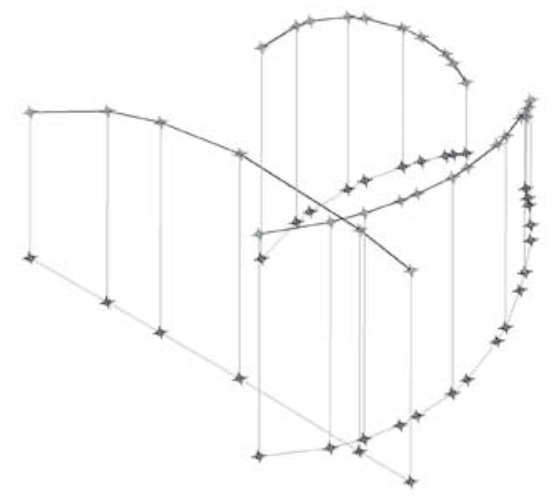

Fig. 13. Curvas obtenidas del análisis de los nervios. Proyección horizontal de las mismas.

la determinada por la proyección de los mismos sobre dicho arco. De este modo se puede conocer con precisión la posición de cada punto de un nervio, que siempre está referida al prinzipalbogen.

Para ilustrar este aspecto, se ha analizado la geometría de tres nervios de la bóveda (fig. 12). Uno de ellos es una curva plana, mientras que los otros dos son curvas espaciales. Tras obtener la posición espacial de cada uno de los puntos de las juntas entre las dovelas de los nervios (fig. 13), se han comprobado dos aspectos. En primer lugar, se observa que la proyección de los nervios curvos tridimensionales sobre el plano horizontal es un arco de circunferencia, es decir, que el trazado de la planta de la bóveda se ha llevado a cabo con líneas rectas y arcos de circunferencia. En segundo lugar (fig. 14), se han tomado las distancias entre los puntos a lo largo de la curva en la que se encuentran, y se han trasladado a una línea horizontal, para después, sobre la vertical de cada uno de ellos, situar la altura a la que se encuentran sobre el suelo. El resultado de situar estos puntos es, en los tres casos, un arco de circunferencia, que no es otro que el prinzipalbogen. Es decir, se

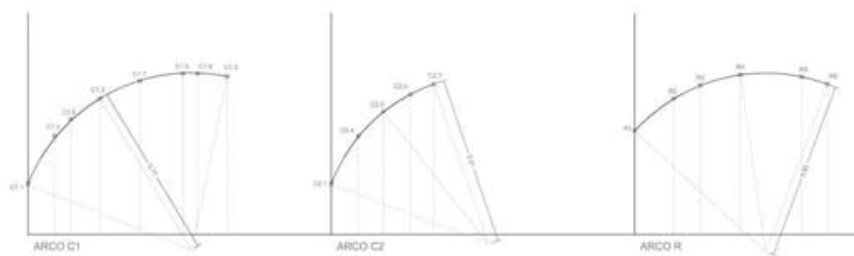

Fig. 14. Situación de los puntos sobre una recta horizontal y a la altura medida en el levantamiento. Comprobación de la posibilidad de que se encuentren sobre un mismo arco de circunferencia.

demuestra que cada punto puede estar definido por este círculo que determina la altura de los puntos en función de la longitud recorrida en la planta. De este modo, se puede definir la geometría de unas líneas curvas espaciales aparentemente arbitrarias. Es este un claro ejemplo de una metodología sistemática para generar formas extremadamente complejas.

8 W. MÜLLER, op. cit., 1990. 


\section{Conclusiones}

El gótico tardío, aquel que se construye a lo largo de los siglos XV y XVI, constituye una corriente que se extiende más allá de sus confines naturales sobre el pleno Renacimiento. Los términos de tardío decadente o manierista son los que con mayor frecuencia se usan para referirse a este periodo y a los maestros que lo protagonizaron. Sin embargo, la belleza, el ingenio y la optimización de medios que subyace tras los abovedamientos del último gótico nos hacen pensar que es precisamente entonces cuando este estilo alcanza en Europa su completa madurez. Todo parece indicar que fue precisamente en su apogeo cuando el gótico fue abandonado por un clasicismo que representaba mejor la vuelta a las raíces grecolatinas europeas.

Desde el punto de vista constructivo, la evolución tecnológica observada está marcada por una tendencia hacia la búsqueda de la estandarización y simplificación de los sistemas. De este modo, al mismo tiempo que los diseños de las bóvedas adquirían caracteres más complejos y elaborados, se desarrollaban técnicas que permitían facilitar el trazado y la construcción de las mismas.

La sistematización de las curvaturas de los nervios supone beneficios claros. De un modo directo, la ejecución de las dovelas se simplifica en cierta medida, ya que todas las piezas son iguales. Sin embargo, las ventajas más significativas están relacionadas con la organización de la obra por un lado (reduciendo las necesidades de numeración y clasificación para el almacenamiento y colocación de las dovelas), y en la transmisión de las órdenes de ejecución desde los maestros a los canteros por otro lado. La traza ideada por el arquitecto debía traducirse en instrucciones para los operarios, y, si bien alguno de los métodos expuestos rompía la integridad geométrica de los arcos, la igualación de curvaturas suponía una gran ventaja de cara a esta transmisión de órdenes. De este modo, la geometría conceptual del proyecto se adaptaba a la realidad práctica de la obra, puesto que las diferencias eran inapreciables a simple vista. Todavía más necesarios eran los métodos como el prinzipalbogen centroeuropeo, dada la complejidad geométrica de estas obras (con curvas espaciales), que imposibilitaba el trazado de elevaciones de arcos a escala para la talla de dovelas o la construcción de cimbras.

La visión peyorativa que la historiografía tradicional ha mostrado sobre este gótico se contradice con el formidable éxito del que gozó en casi toda Europa. Si además se considera el sofisticado manejo de los recursos geométricos y los notables avances tecnológicos que se produjeron, no es exagerado considerar que los abovedamientos bajomedievales constituyen una de las páginas más brillantes de la historia de la construcción europea. 